\title{
cTBS over contralesional homologue areas deteriorates speech output in isolated Apraxia of Speech after stroke
}

Brigitte C. Kaufmann ${ }^{1 \text { a,b }}$, Manuela Pastore-Wapp ${ }^{1 \text { b, c }}$, Maria Lübeck ${ }^{b}$, Monica Koenig ${ }^{b}$, Stephan Bohlhalter ${ }^{\mathrm{b}}$, Tim Vanbellingen ${ }^{\mathrm{b}, \mathrm{d}}$, Dario Cazzoli ${ }^{\mathrm{a}, \mathrm{d}}$, Thomas Nyffeler ${ }^{\mathrm{a}, \mathrm{b}, \mathrm{d}^{*}}$

${ }^{1}$ contributed equally

${ }^{\text {a }}$ Perception and Eye Movement Laboratory, Department of Neurology, University of Bern, Switzerland,

${ }^{\mathrm{b}}$ Neurocenter, Luzerner Kantonsspital, Switzerland

${ }^{\mathrm{c}}$ Support Center for Advanced Neuroimaging (SCAN), Institute of Diagnostic and Interventional Neuroradiology, Inselspital, University Hospital, University of Bern, Switzerland ${ }^{\mathrm{d}}$ Gerontechnology and Rehabilitation Group, ARTORG Center for Biomedical Engineering Research University of Bern, Switzerland

\footnotetext{
*Address for correspondence: Prof. Thomas Nyffeler, ARTORG Center for Biomedical Engineering Research, University of Bern, Bern, Switzerland.

E-mail address: thomas.nyffeler@luks.ch
} 
Apraxia of speech (AOS) is an impairment of motor speech planning/programming, characterized by a combination of phonemic segmental changes and articulatory distortions [1]. AOS is most often accompanied by aphasia, whereas isolated forms may rarely occur after focal damage to the left precentral gyrus [1]. How AOS recovers after brain damage is poorly understood [2]. In particular, it is not known whether recovery of AOS solely depends on the functional reorganisation of perilesional areas [3, 4], or whether it also depends on the compensation through contralesional homologue areas. Based on findings from healthy subjects showing that speech production is controlled by both hemispheres [2], it may be hypothesized that the contralesional homologue areas contribute to recovery of AOS.

We had the exceptionally rare opportunity to test this hypothesis, in a patient that partially recovered from isolated AOS due to a small subacute stroke of the left precentral gyrus (time interval stroke to testing 24 days). We used continuous Theta Burst Stimulation (cTBS) to transiently inactivate the contralesional homologue areas, and therefore act as a transient 'virtual lesion'. If the hypothesis would be true then inactivating an area that participates as a substrate in mediating functional recovery should uncover the original deficit in a patient who has partially recovered. However, if the hypothesis of a solely cortical reorganization adjacent to the lesion would be true, inactivation of contralesional areas should not uncover the original deficit.

A.G., a 69-year-old, right-handed, Swiss-German native speaker woman suffered from isolated AOS due to a first, small ischemic stroke of the left precentral gyrus (Figure 1a). On the Apraxia of Speech Rating Scale, she showed moderate to severe AOS (8 points). Five out of six primary distinguishing AOS features were present in her speech: distorted sound substitutions and additions, increased sound distortions with increased utterance length, increased speech rate and inaccurate speech alternating motion rates in rapid syllable repetition. She had no buccofacial apraxia, no limb apraxia, and no overt aphasia, except for a minor comprehension deficit for reversible, non-canonical sentences (Figure 1c). Within the first three weeks she partially recovered from AOS. A.G. gave written informed consent prior to participation. The study was performed according to the Declaration of Helsinki.

To evaluate whether the contralesional homologue areas are involved in AOS recovery, one continuous train of cTBS, an inhibitory NIBS protocol with after-effects lasting approximately 20 minutes [5], was applied with a round coil (outer radius of $60 \mathrm{~mm}$, Magnetic coil Tranducer, MC-125, Medtronic) in a sham-controlled, double-blind approach over FC6, which, according to the 10-10 EEG system, overlies the right precentral gyrus [6]. The cTBS train comprised of 267 bursts, each consisting of 3 pulses at $30 \mathrm{~Hz}$, repeated at $6 \mathrm{~Hz}[5]$, 
resulting in a total duration of $44 \mathrm{~s}$. cTBS was performed 24 days after stroke, and was delivered at $80 \%$ of the patient's resting motor threshold; sham stimulation was applied with a sham coil (Magnetic Coil Transducer MC-P-B70) one week later.

A speech-testing protocol was performed before and immediately after each stimulation condition, comprising following tasks: (1) a sequential motion rate (SMR) - word repetition task, in which three words had to be repeated five times, as fast as possible; (2) the readingaloud of minimal pairs; hereby, A.G. had to read aloud eight word pairs that differ in only one phonological element. This task places high demands on motor planning and programming abilities, in order to achieve phoneme accuracy in articulatory contrasts [7]; (3) a speech production task, in which the patient had to read aloud a short fable (110 words), to assess her consecutive performance. Hence, in all tests, the patient spelled 141 words.

All tests were video-recorded and rated by two independent, blinded language therapists. Hereby, each word was separately rated as correct or incorrect. Words were rated as incorrect if at least one of the following errors occurred: segmental errors (sound distortions, distorted sound substitutions or additions, exaggerated sound prolongations) or prosodic errors (intra/intersyllabic pauses). For each task, the total number of correct and incorrect words was computed. Incorrect words were classified into prosodic or segmental errors. In addition, the differences between cTBS and sham stimulation were calculated (error rate in \%).

Before stimulation, the total number of speech errors was similar in the cTBS (total error rate: 56) and in the sham (total error rate: 54) conditions. After cTBS, the speech of the patient deteriorated (total error rate: 69; predominantly attributable to errors in the segmental category; Figure 1 B) and the patient spontaneously complained about a worsening of speaking. In contrast, after sham stimulation, the speech rate improved (total error rate: 37 ), probably due to increased arousal and a re-test effect. The difference between the two conditions was 32 errors or an error rate of $22 \%$ (Figure 1C).

In a patient that partially recovered from isolated AOS due to a small subacute stroke of the left precentral gyrus, inhibition of the right precentral gyrus by means of NIBS was shown to temporarily deteriorate speech output compared to sham stimulation. This suggests that recovery of motor speech planning, which is lateralized to the left hemisphere [8], depends, at least partially, on the function of contralesional homologue areas. Our findings are also in line with results obtained in healthy subjects, showing that motor speech production, although lateralized towards the dominant hemisphere, relies on motor cortical networks of both hemispheres [2]. Regarding the future of AOS therapy, our results further suggest that inhibition of 
contralesional homologue areas should probably be avoided. This is in contrast to NIBS application in patients with aphasia, where based on the principles of interhemispheric inhibition, inhibitory stimulation over the contralesional right homologues may improve language function [9]. In addition, although successful on a clinical level, a bihemispheric NIBS approach with excitation of the left perilesional area and inhibition of contralesional right homologues [10] could possibly be less efficient than solely excitatory NIBS protocols, applied over the perilesional tissue $[3,10]$.

\section{DECLARATION OF COMPETING INTERESTS}

None

\section{FUNDING}

This work was supported by the Swiss National Science Foundation SNF Grants \#320030 and $\# 155954$. 

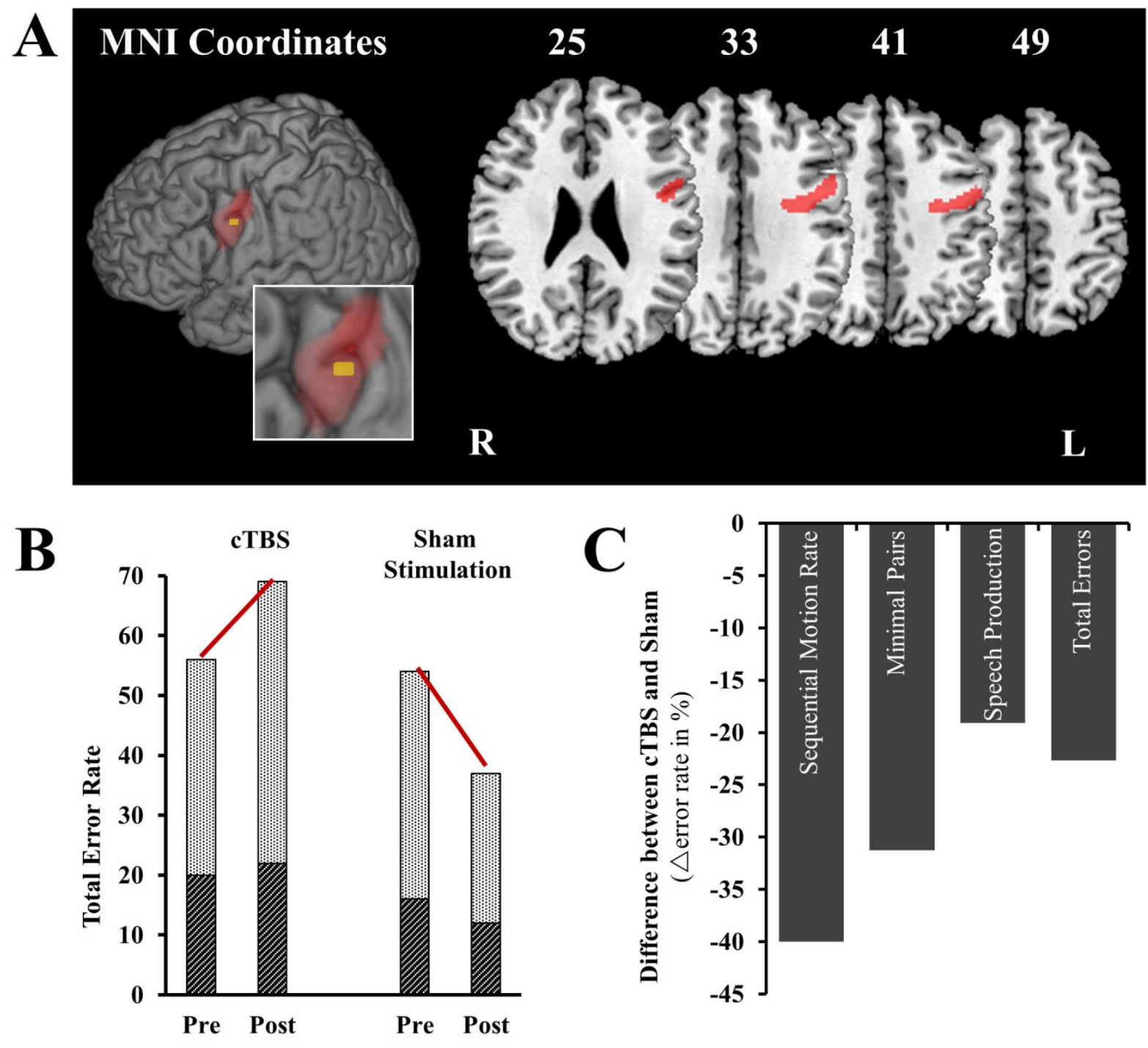

Prosodic Errors ; Segmental Errors

\begin{tabular}{clr}
\hline & Token Test (short version) & $10 / 10$ \\
& Word repetition & $20 / 30$ \\
& Object naming & $30 / 30$ \\
Aachener Aphasie Test (AAT) & Situation/ Action naming & $22 / 30$ \\
& Auditive word comprehension & $27 / 30$ \\
& Auditive sentence comprehension & $30 / 30$ \\
& Written word comprehension & $30 / 30$ \\
& Written sentence comprehension & $24 / 30$ \\
\hline \multirow{2}{*}{ Boston Diagnostic Aphasia } & Goodglass Written Text Comprehension & $9 / 9$ \\
\hline Examination (BDAE) & & $39 / 40$ \\
\hline \multirow{2}{*}{ Writen Text Comprehension } & sentences with canonical word order & $18 / 40$ \\
\hline & (e.g. «Das Mädchen wirft dem Jungen den Ball») & Sentences with non-canonical word order (e.g. «Dem \\
\hline
\end{tabular}

${ }^{a}$ score deduction due to self-corrections 
Figure 1. (A) Normalised high resolution MRI scan of patient A.G., showing an isolated ischemic stroke in the left precentral gyrus (red). Damage to the left precentral gyrus has been previously shown to be associated with AOS ([1], significant cluster highlighted in yellow). The lesion was manually delinated on A.G.'s high-resolution MRI scan, normalised and represented on the $\mathrm{CH} 2$ template, as available in MRIcron (http://www.mccauslandcenter.sc.edu/crnl/chris-rordens-neuropsychology-lab). Axial slices are oriented according to the neurological convention. (B) Speech performance of the patient, measured as the total number of errors pre and post cTBS and Sham stimulation, respectively. Prosodic errors are shown in dark grey, segmental errors in light grey. The change in the total number of errors between pre and post stimulation conditions is highlighted in red. (C) Difference in Speech perfromance between sham and cTBS stimulation (in \%) calculated for the three subtests of the speech-testing protocol (sequential motion rate, reading-aloud of minimal pairs, speech production). Comparing the total error rate between cTBS to sham stimulation, a difference of $22 \%$ was found. (D) Individual raw scores of the baseline language screening. 


\section{REFERENCES}

[1] Itabashi R, Nishio Y, Kataoka Y, Yazawa Y, Furui E, Matsuda M, Mori E. Damage to the Left Precentral Gyrus Is Associated With Apraxia of Speech in Acute Stroke. Stroke 2016; 47: 31-36. doi: 10.1161/STROKEAHA.115.010402

[2] Terao Y, Sakurai Y, Ugawa Y. Letter by Terao et al Regarding Article "Damage to the Left Precentral Gyrus Is Associated With Apraxia of Speech in Acute Stroke". Stroke 2016; 47. doi: 10.1161/STROKEAHA.116.012755.

[3] Maltifano C, Rossetti AC. Malloggi C, Scarano S, Tesio L. rTMS can improve poststroke apraxia of speech. A case study. Brain Stimulation 2018. doi: 10.1016/j.brs.2018.12.006.

[4] Marangolo P, Marinellib CV, Bonifazia S, Fioria V, Ceravoloa MG, Provincialia L, To maiuolod F. Electrical stimulation over the left inferior frontal gyrus (IFG) determine long-term effects in the recovery of speech apraxia in three chronic aphasics. Behavioural Brain Research 2011; 225. doi:10.1016/j.bbr.2011.08.008.

[5] Nyffeler T, Cazzoli D, Wurtz P, Luthi M, von Wartburg R, Chaves S, Deruaz A, Hess CW, Muri RM. Neglect-like visual exploration behaviour after theta burst transcranial magnetic stimulation of the right posterior parietal cortex. Eur J Neurosci 2008; 27;7;180913. doi: 10.1111/j.1460-9568.2008.06154.x .

[6] Koessler L, Maillard L, Benhadid A, Vignal JP, Felblinger J, Vespignani H, Braun M. Automated cortical projection of EEG sensors: Anatomical correlation via the international 10-10 system. NeuroImage 2009; 46;1; 64-72. doi: 10.1016/j.neuroimage.2009.02.006

[7] Wambaugh JL, Doyle PJ, Kalinyak MM, West JE. A minimal contrast treatment for apraxia of speech. Clinical Aphasiology 1996; 97-108.

[8] Lotze M, Seggewies G, Erb M, Grodd W, Birbaumer NT. The representation of articula tion in the primary sensorimotor cortex. Neuroreport 2000; 11; 2985-2989.

[9] Otal B, Olma MC, Flöel A, Wellwood I. Inhibitory non-invasive brain stimulation to ho mologous language regions as an adjunct to speech and language therapy in post-stroke aphasia: a meta-analysis.Front Hum Neurosci 2015; 9; 236. doi: 10.3389/fnhum.2015.00236.

[10] Marangolo P, Fiori V, Cipollari S, Campana S, Razzano C, Di Paola M, Koch G, Caltagirone C. Bihemispheric stimulation over left and right inferior frontal region enhance es recovery from apraxia of speech in chronic aphasia. European Journal of Neuroscience 2013; 38; 3370-3377. doi:10.1111/ejn.12332. 